\title{
Presentación
}

\section{Turismo y centros históricos: un dossier candente}

\section{Daniel Hiernaux-Nicolas*}

La oportunidad que se ofreció a los autores de los textos que integran el dossier principal de este número de Estudios Críticos del Desarrollo, abre la puerta para introducir temas que no son con frecuencia asociados con los estudios del mismo. Sin embargo, no cabe duda de que las ciudades se han vuelto no sólo los nodos primordiales a partir de los cuales se definen y manejan las nuevas orientaciones de la economía mundial, sino que además son lugares en los que los procesos de acumulación son sumamente importantes y complejos (Sassen, 2007).

La financierización creciente de la economía capitalista, por ejemplo, no sólo se despliega desde los centros financieros ubicados en el corazón de las ciudades, sino que, a la vez, transforma de modo radical las urbes (de Mattos, 2016). La expansión de la industria de la construcción, dirigida cada vez más por empresas multinacionales gigantes como Odebrecht, entre otras, revela que la producción del espacio urbano se ha vuelto un campo particularmente propicio para una acumulación acelerada. Una simple mirada a la edificación de nuevas instalaciones hoteleras manejadas por operadoras (cadenas) por todo el mundo revela a qué grado se ha aprovechado el turismo como esfera de acumulación a través de la edificación de estructuras gigantescas.

A su vez, la construcción de infraestructuras multimillonarias como trenes rápidos para enlazar ciudades, autopistas urbanas y aeropuertos

* Docente investigador de la Facultad de Ciencias Políticas y Sociales de la Universidad Autónoma de Querétaro. Correo-e: danielhiernaux@gmail.com 
nuevos apuntan en la misma dirección y a la privatización del espacio y de los soportes materiales urbanos (Márquez y Pradilla, 2017). El consumo desenfrenado impulsado por las empresas de distribución y mediante las facilidades de crédito que otorgan los organismos financieros ha impulsado, además, la construcción de centros comerciales a lo largo del planeta, con un crecimiento inusitado del número de equipamientos y de la escala de los mismos.

Finalmente, la expansión de las periferias a partir de la construcción de viviendas unifamiliares, de nuevo con la bendición conjunta de los gobiernos neoliberales y de los fondos financieros — fondos de pensiones, entre otrosha propiciado que la industria de la construcción sea vista como uno de los sectores más dinámicos de la economía mundial (Santana y Alzate, 2017).

Hasta ahora nos hemos referido a espacios nuevos o a la recuperación de espacios en desuso por la crisis del fordismo, como es el caso de las zonas portuarias en algunas ciudades — por ejemplo, Puerto Madero en Buenos Aires - o de antiguos parques o zonas industriales de empresas medianas y pequeñas — como el «Nuevo Polanco» en la Ciudad de México sobre terrenos antes usados por una industria hoy desaparecida o relocalizada. Ciertamente, de esta manera, las ciudades se están adecuando de manera rápida y violenta en pocas décadas al modelo de acumulación del neoliberalismo, con un ritmo de transformaciones inusitado que merece remitir a Marx con su expresión «todo lo sólido se desvanece en el aire», puesta de moda desde tiempo atrás por Marshall Berman (1988). Sin embargo, si una parte de lo que se desvanece se vuelve «líquido», como lo ha sustentado con fuerza y de manera convincente Zygmunt Bauman (2007), no es menos cierto que mucho de lo sólido/tradicional se desvanece bajo las picotas para emerger nuevamente como estructuras materiales sólidas, como es el 


\section{PRESENTACión}

caso de las áreas en reconversión, aun si las actividades recientes no tienen nada que ver con el pasado.

Esta transformación de las ciudades hacia una nueva modernidad diseñada desde la acumulación y las finanzas esculpe una ciudad marcada por la verticalidad del paisaje, la homogeneización de los modelos urbanos a lo largo del mundo y su evidente tecnificación reflejada en la voluntad de volver las ciudades «inteligentes» (Schiavo y Gelfuso, 2017), es decir, sometidas a la presión de las grandes empresas de la era digital que desean ensanchar sus mercados en las ciudades a través de nuevos diseños para las mismas y una gestión tecno-política enmarcada en un nuevo modelo de régimen urbano, eso de manera independiente del color político del régimen que dirige la ciudad.

Los centros de las ciudades actuales parecen haberse vuelto el locus de las discusiones más acérrimas sobre el futuro de las ciudades. No existe una separación clara entre «críticos» y «neoliberales», sino una confluencia de posiciones epistemológicas y políticas del devenir de los centros históricos que no ha desembocado en una teoría clara acerca de lo que está ocurriendo en los mismos. No cabe duda de que esta situación no es exclusiva de los países del llamado «Norte», sino que se extiende a lo que identificamos como «el Sur». Inclusive y paradójicamente, algunos países de regímenes políticos herederos del comunismo como Cuba, China y Vietnam se enfrentan a semejantes transformaciones de sus áreas centrales.

Los autores del presente número de Estudios Críticos del Desarrollo se han planteado proponer una serie de reflexiones sobre uno de los procesos reconocido como transformador intensivo de los centros de ciudades: el turismo urbano-cultural en ciudades mexicanas. Esta modalidad de hacer turismo, bien se sabe que se diferencia singularmente del turismo de 
las tres «S»: «sea-sand-sun» (mar, arena y sol) que dominó la historia del turismo en México. Al desempeñarse en espacios habitados por residentes permanentes — en ocasiones con una vida urbana intensa-, y cumplir con deseos e intereses diferenciados de las prácticas socioespaciales de estos residentes en su vida cotidiana, los turistas trastornan esa vida activa en el día a día de sus prácticas turísticas. A la par, modifican radicalmente algunos aspectos de la mecánica urbana como es el mercado inmobiliario, lo que favorece un incremento de alquiler, la tendencia a la venta de inmuebles para destinarlos a fines turísticos, y la modificación de usos, por ejemplo por rentas de corto plazo en tratos directos entre el dueño y el turista a través de plataformas informáticas con un alto grado de ilegalidad.

Para calificar estos procesos de transformación se han acuñado neologismos como patrimonialización, turistificación, gentrificación, los cuales han sido adoptados con éxito diferencial y han sido objeto de fuertes polémicas entre las diversas corrientes epistemológicas que se han interesado en el objeto de estudio «Centro Histórico» (Hiernaux, 2016; Hiernaux y González, 2014).

Vale señalar que los antiguos centros de las ciudades no están totalmente exentos de intervenciones del capitalismo moderno, ni siquiera de edificaciones que marcan el prestigio de alguna empresa o tycoon del capitalismo actual. Sin embargo, las políticas de protección patrimonial puestas en práctica por los gobiernos locales y nacionales han impedido que se prosigan e intensifiquen las degradaciones de los centros, en particular intensas en la época de la modernidad triunfante.

Por otra parte, el consumismo imperante en las sociedades actuales ha propiciado que no sólo se realice un consumo creciente de bienes de uso personal (comida, vestimenta, adornos, muebles, vehículos) que genera 


\section{Presentación}

desechos, sino que las poblaciones con cierto capital social y cultural, y por supuesto económico, adopten consumos simbólicos sustanciales que les permite marcar cierta distinción bourdieusiana frente al resto de la población cada vez más masificada y homogeneizada (Bourdin, 2007).

El turismo permite efectuar semejante consumo valorizado por medio de la marca asociada a un destino, una instalación, un festival, etcétera. En este contexto, un Centro Histórico resulta ser entonces un acervo insospechado de elementos materiales e inmateriales susceptibles de ser consumidos por locales y ajenos.

No es de extrañar que las políticas de desarrollo local aplicadas a los centros se identifiquen plenamente con el llamado internacional (en concreto de la Organización de las Naciones Unidas para la Educación, la Ciencia y la Cultura, UNESCO, y de la Organización Mundial del Turismo, OMT) a articular patrimonio y turismo como una nueva clave para impulsar la economía urbana, como ya lo había anunciado Michael Porter años atrás (Porter, 1995). Si bien se suele señalar que los procesos de turistificación tienden a homogeneizar los centros, mostrándose significativas similitudes en los efectos del turismo y también en los paisajes urbanos, no es menos cierto que diversas condiciones provocan que cada centro muestre resultados distintos.

En ocasión de este número se han invitado estudiosos de varias ciudades medias en las que se observan procesos de cambio relevantes a partir del desarrollo del turismo en sus centros históricos: se trata de Zacatecas (caso presentado por Guadalupe Margarita González Hernández), Querétaro (trabajo de Daniel Hiernaux-Nicolas y Carmen Imelda González Gómez), Mérida (investigación realizada por José Humberto Gómez Fuentes y Diana María Magnolia Rosado Lugo) y Oaxaca (analizado por Mabel Yescas Sánchez). El caso de la Ciudad de México, desarrollado por 
Víctor Delgadillo, es un referente imprescindible porque parecería ser, como lo sugiere la lectura del texto, que se anticipa a situaciones que podrán verificarse en breve en las ciudades medias estudiadas.

Como se acaba de afirmar, la introducción del turismo en los centros mencionados es el mayor proceso de cambio que se ha observado en los últimos años. Lo que Marie-Françoise Lanfant (1994) ha bautizado como «turistificación» es de hecho la puesta en turismo de espacios construidos o públicos, de eventos y de costumbres antes destinados a otros usos o referidos a otras prácticas, como las religiosas. La turistificación es en parte una recuperación de ciertos aspectos tradicionales de los centros de las ciudades: fiestas religiosas, uso de los espacios públicos, movilidad peatonal, edificios públicos. Para algunos de los centros estudiados, esta turistificación de lo tradicional es algo que se ha dado desde décadas atrás, como es el caso de Oaxaca, destino apreciado de larga data por mexicanos y extranjeros; otros, como Querétaro, sólo en fechas recientes se han visto introducidos en el turismo.

No obstante, la turistificación además de ser un proceso de recuperación de lo existente, es una reconfiguración de lo mismo a través de la introducción de nuevas prácticas y nuevos usos. Bien se sabe, por ejemplo, que la Guelaguetza de Oaxaca es relativamente reciente. De esa manera, elementos materiales como construcciones y prácticas sociales se han visto absorbidos por una lógica de comercialización para el uso de los turistas (González, 2014).

Es pertinente mencionar que si bien se usa la palabra «turista» en un sentido general, estrictamente se está hablando de diversas clases de visitantes incluyendo los mismos residentes de la ciudad estudiada, personas que vienen a pasar el día y que provienen de otras localidades y, finalmente, turistas en stricto sensu, es decir, aquellos visitantes que pernoctan en la localidad, sean nacionales o internacionales. Se entiende así que se 


\section{PRESENTACIÓN}

mezclan prácticas de ocio con aquellas que son meramente turísticas y resulta cada vez más complejo distinguirlas. En ese sentido, el investigador David Crouch indica, con toda razón, que sería mejor hablar de leisure/tourism (ocio/turismo) en vez de «turistas» para revelar la complejidad de las prácticas realizadas por visitantes de distintas categorías (Crouch, 1999). En el contexto del presente trabajo, tanto el autor de estas líneas como los que analizan los distintos centros estudiados, usan el término «turistas» para referirse a la categoría mixta de turistas y demás visitantes. ${ }^{1}$

Zacatecas es un caso relevante para documentar algunos de los aspectos expuestos más arriba: la intención del escrito de Guadalupe Margarita González Hernández es reconstruir lo que denomina la genealogía de la mercantilización del patrimonio de la capital del estado zacatecano. Evidencia que el proyecto liderado por el gobierno local tiene sus raíces en unas primeras intervenciones en la década de 1980, aun si admite que fue la declaración de pertenencia al Patrimonio Cultural de la Humanidad la que volvió a acelerar un proyecto de turistificación que se había hecho lento. El repunte de inversiones que documenta a partir de 1993 y hasta 2006 implicó gastos superfluos y, sin duda, generadores de exclusión de la población más desprotegida del Centro Histórico.

A la vez, afirma tajantemente y comprueba lo que se ha sostenido en diversos estudios: el turismo genera poca riqueza, salvo para los dueños de los establecimientos ligados al ocio y al turismo, mientras que los trabajadores se mantienen en actividades precarias, mal pagadas, con contratación efímera.

${ }^{1}$ Inclusive falta señalar que los residentes de larga duración cuyo análisis es central en el estudio de Mérida, se suelen clasificar como «turistas residenciales» aunque son residentes de pleno derecho aunque de presencia no permanente. 
En años recientes, un movimiento social se constituyó con diversas posturas y grupos en contra de algunas de las acciones del Estado asociado con grupos de capital, entre los cuales la cervecería Modelo que iba a recibir la concesión del conocido Mercado González Ortega para transformarlo en una suerte de bar, a la usanza con seguridad del Mercado San Miguel de Madrid, un modelo reconocido en ese sentido. Si bien la movilización social permitió repensar o contrarrestar algunas iniciativas, se transformó en una lucha en el seno del espacio político local. La autora concluye que estas transformaciones, lejos de alcanzar los objetivos supuestamente planteados con relación a un posible auge del Centro Histórico, más bien han generado mayor exclusión social, la mercantilización del patrimonio y un escaso desarrollo de la actividad turística.

Querétaro es un caso relativamente diferente, pero apunta a tendencias similares. En una primera parte del ensayo, Daniel Hiernaux-Nicolas y Carmen Imelda González Gómez se plantean la pregunta de saber si la ciudad de Querétaro y en particular su Centro Histórico se encuentran envueltos en un proceso de gentrificación. Los autores señalan que el trabajo de campo realizado muestra que aunque se observa un cierto desplazamiento de la población tradicional, es significativo que las transformaciones del centro y en particular del Barrio de la Cruz donde efectuaron su trabajo de campo se derivan sobre todo de las políticas públicas mediante intervenciones patrimoniales, regeneración del espacio público (calles y diversas plazas en todo el centro) e impulso de los negocios ligados al ocio y al turismo, de esa manera se confirma lo que se observa en Zacatecas.

Indican que el imaginario patrimonialista, impulsado desde la UNESCO y asumido por las autoridades mexicanas desde el nivel más alto, ha propiciado la mercantilización del Centro Histórico y, por ende, su turistificación 


\section{Presentación}

creciente. A ello se asocia, según los autores, un proceso de «higienismo urbano» no muy diferente del que se ejerció en diversas partes del mundo en el siglo XIX para «dignificar» el espacio central de la ciudad, lo que ha conducido, como bien se puede imaginar, a una creciente elitización del centro y a una exclusión de los más pobres, entre los cuales resaltan las mujeres indígenas que tradicionalmente vendían sus artesanías —en especial la conocida muñeca de Amealco - en las calles y en las plazas del centro. Se observa además un éxito importante y un crecimiento evidente de la capacidad de recepción de turistas — incluyendo el modelo Airbnb ampliamente criticado en Europa-, que conforta a las autoridades en su proyecto excluyente y modernizador. Un aspecto importante es notar que el desplazamiento de la población no es tan evidente como lo puede ser en otros lugares, en particular, como lo demuestra su trabajo de campo, por la alta tasa de propiedad de la vivienda, tema que invalida para los casos mexicanos, la afirmación tajante de los autores anglosajones reconocidos sobre la gentrificación (por ejemplo, Smith, 1996; Lees et al., 2005 y 2007).

A pesar de que la investigación sobre Zacatecas evidencia cierta reacción de la población a las transformaciones de las áreas centrales y aquella sobre Querétaro menciona una reacción incipiente a esos cambios, para el caso de Oaxaca, la crisis social de la localidad y el estado que se manifiesta de manera reiterada en el espacio central, es un factor decisivo para explicar el devenir del Centro Histórico y el eventual éxito o fracaso de las políticas públicas. En su aportación, Mabel Yescas Sánchez identifica los actores que intervienen en la escena del Centro Histórico. Demuestra que la promoción del turismo en la capital oaxaqueña se remonta a los años 1930, en parte por la introducción del ferrocarril pero sobre todo a partir de una política deliberada de apoyo a una localidad devastada por fuertes sismos. 
De particular interés es el hecho de que la recuperación del Centro Histórico oaxaqueño se ha hecho de la mano de un empresario reconocido a escalas nacional e internacional, Alfredo Harp Helú, considerado por unos como mecenas y por otros como cacique local. Las obras emprendidas por Harp Helú, entre las que destaca la recuperación total del convento de Santo Domingo, han sido esenciales para reactivar el centro, cuya actividad comercial y turística fue drásticamente afectada por diversos movimientos sociales, en específico el de 2006. La autora constata que las políticas públicas y las intervenciones privadas van de la mano, con una clara tendencia a no favorecer los intereses de los habitantes.

El texto anterior muestra, además, la relevancia que puede llegar a tener la presencia de un personaje de alto capital económico en la definición de las políticas sobre un Centro Histórico, situación que se volverá a presentar en el caso de la Ciudad de México. Muestra a la vez que las políticas de turistificación y patrimonialización pueden generar efectos contradictorios para la población local, en concreto cuando se está frente a condiciones de marginación elevada y de pobreza, no sólo en la ciudad sino en todo el estado, lo que se refleja también en la presencia permanente de ambulantaje en el centro que compite por el uso del espacio público con el turismo.

Mérida, analizada por José Humberto Fuentes Gómez y Diana María Magnolia Rosado Lugo, es otra de las ciudades medias que se enfrenta a la turistificación. Sin embargo, en este aporte, los autores llaman la atención del lector sobre una dimensión muy importante y escasamente estudiada, la relación entre turistas, nuevos residentes de mayor duración —en su mayoría extranjeros - y residentes tradicionales.

Según los autores, a lo largo de los años se ha dado una fuerte degradación de algunas partes del Centro Histórico, lo que ha llevado al abandono 


\section{Presentación}

de edificios y a la destrucción de un patrimonio considerable. Esto como resultado de una expansión significativa del comercio y del transporte en una ciudad en pleno crecimiento hacia su periferia que provocó que los cuarteles centrales de la ciudad se encuentren en la actualidad poco habitados, inclusive con calles enteras vacías.

Sin embargo, desde un par de décadas, calles enteras han sido reactivadas por extranjeros (los autores estiman a partir de datos concretos que éstos son propietarios de casi un cuarto de los predios del centro), quienes han invertido en una remodelación de viviendas que ha ocasionado problemas de convivencia entre meridianos y nuevos residentes. $\mathrm{La}$ declaración de Mérida como Capital Americana de la Cultura en 2000, fruto de un intenso trabajo de lobby de las autoridades, llamó la atención internacional sobre la capital de Yucatán y resultó en un flujo creciente de visitantes y de residentes temporales en la época de menor calor, los cuales regresan a sus lugares de origen en los meses de mayores temperaturas. Así, se ha repoblado en parte el centro, pero esta nueva población residente mostró su desagrado frente a la proliferación de bares y antros diversos que generan ruido y afectan la «paz» residencial. Situación que se ha podido observar de manera álgida en Querétaro, pero también en ciudades como Barcelona (en la Ciudad Vieja) o en París en el entorno del Museo Centro Pompidou, entre otros lugares.

El dossier ofrece al final un texto muy significativo de Víctor Delgadillo. La pregunta central que se hace el autor es saber si el medio siglo de intento de rescatar el Centro Histórico de la Ciudad de México ha sido realizado a favor de sus habitantes o si apunta a la formación de un parque temático para visitantes y nuevos residentes de mayor ingreso. El especialista empieza por hacer un recuento pormenorizado de las acciones de 
rescate del centro, para enseguida introducir el tema del turismo. Éste ha conocido un crecimiento muy relevante que va de la mano con las mejorías efectuadas en las infraestructuras, el rescate patrimonial y una política de seguridad pública que implica no sólo mayor vigilancia por el personal policiaco y por un extenso sistema de videocámaras, sino también los desalojos permanentes de personas «indeseables» que viven o acuden al centro como prostitutas, vendedores ambulantes, limosneros y demás, efectuados de manera generalmente violenta.

De nuevo, este trabajo muestra el papel desempeñado por un tycoon, en este caso el conocido multimillonario Carlos Slim, quien trabajó junto con el gobierno local desde 2000 para promover un cierto modelo de renovación y rescate del centro, que el autor califica muy justamente de «nuevo orden urbano» para el Centro Histórico. Un aspecto novedoso y digno de mayor investigación es la anotación que hace el autor de que parte de los beneficiarios de vivienda en el plan de Slim y de las autoridades para volver a poblar el centro de la capital — muy despoblado — ha preferido, últimamente, colocar su vivienda en el mercado de la renta turística, tipo Airbnb, inclusive se volvió propietario de nuevas viviendas para integrarlas a ese jugoso mercado.

Es un tema que no se ha estudiado a profundidad y que merecería la atención de los investigadores por las profundas repercusiones que afectan el mercado inmobiliario, que empujan a la alza las rentas y transferencias de propiedad y que contribuyen, con toda claridad, a la exclusión de los más desfavorecidos.

Así termina el dossier central de la revista: resulta evidente que no puede quedarse así la investigación de los múltiples temas abordados en los cinco trabajos. A lo largo de los artículos resaltaron diversas problemáticas que 


\section{Presentación}

conviene sintetizar en unos puntos centrales, que además pudieran plantearse como parte de una agenda de investigación sobre los centros históricos mexicanos, que dejo a disposición del lector:

a) Las políticas públicas han propiciado una turistificación de los centros desde tiempo atrás, aunque con agendas y temporalidades distintas según los casos.

b) Estas políticas suelen acompañarse de una suerte de «higienismo» social, que evoca las políticas de décadas anteriores, en específico del siglo XIX, mismas que contribuyen a fomentar una elitización de los centros.

c) Bajo diversos mecanismos se advierte la llegada de personas con mayor capital cultural y económico a los centros, quienes pueden volverse a su vez microempresarios turísticos en el marco de la expansión del alojamiento turístico propagado por las redes sociales.

d) En ciertos casos se observa la presencia de personajes relevantes de la vida económica y social del país que intervienen para este rescate. Valdrá la pena analizar la validez y los intereses detrás de esas intervenciones, a la par de investigar la relación entre funcionarios e inversionistas (inmobiliarios o impulsores de actividades de ocio y turismo) que parecen comportarse en conjunto a la manera de un «régimen urbano» específico para los centros históricos.

e) Todo lo anterior puede o no generar movimientos sociales específicos de rechazo a las políticas de turistificación y patrimonialización, sin olvidar el tema de las protestas sociales más amplias (como en el caso oaxaqueño) que pueden llegar a detener los mencionados procesos.

f) Se generaliza la conclusión de que todos los procesos estudiados en sus diversas facetas y contextos, obedecen a tendencias globales y, al mismo tiempo, se instituyen en una suerte de un «nuevo orden urbano» 


\section{DANIEL HiERnAUX-Nicolas}

para los centros, del cual es imprescindible analizar la articulación con las políticas de periferización y modificación de la centralidad de las metrópolis actuales.

\section{Referencias}

Bauman, Zygmunt (2007), Tiempos líquidos. Vivir en una época de incertidumbre, México, Tusquets Editores.

Berman, Marshall (1988), Todo lo sólido se desvanece en el aire. La experiencia de la modernidad, Madrid, Siglo XXI.

Bourdin, Alain (2007), La metrópoli de los individuos, Puebla, Benemérita Universidad Autónoma de Puebla.

Crouch, David (1999), Tourism/leisure/tourism geographies. Practices and geographical knowledge, Londres, Routledge.

De Mattos, Carlos A., (2016), «Financiarización, valorización inmobiliaria del capital y mercantilización de la metamorfosis urbana», Sociologías, 18(42), pp. 24-52.

González Hernández, Guadalupe Margarita (2014), Circo sin pan. Regeneración y mercantilización en el Centro Histórico, México, Miguel Ángel Porrúa/Universidad Autónoma de Zacatecas.

Hiernaux, Daniel (2016), «La gentrificación «criolla` en México: entre el tipo ideal y las prácticas socio-espaciales en los centros históricos mexicanos», en Yazna Contreras, Thierry Lulle y Oscar Figueroa (eds.), Cambios socioespaciales en ciudades latinoamericanas, ¿procesos de gentrificación?, Bogotá y Santiago, Universidad Externado de Colombia/Universidad de Chile, pp. 39-59.

Hiernaux, Daniel y Carmen Imelda González (2014), «Turismo y gentrificación: pistas teóricas para su interpretación», Revista Norte Grande (58), pp. 55-70. 


\section{PRESENTACIÓN}

(2017), La ciudad latinoamericana a debate. Perspectivas teóricas, Querétaro, Universidad Autónoma de Querétaro.

Lanfant, Françoise (1994), «Identité, mémoire et la touristification de nos sociétés»,

Sociétés Revue des Sciences Humaines et Sociales (46), pp. 433-439.

Lees, Loretta, Tom Slater y Elvin Wyly (2007), Gentrification, Londres, Routledge. Lees, Loretta, Hyun Bang Chin y Ernesto López Morales (comps.) (2015), Planetary gentrification, Cambridge, Polity Press.

Márquez, Lisett y Emilio Pradilla (2017), «La privatización de lo urbano», en Daniel Hiernaux y Carmen Imelda González (comps.), La ciudad latinoamericana a debate. Perspectivas teóricas, Querétaro, Universidad Autónoma de Querétaro, pp. 57-92.

Porter, Michael (1995), «The competitive advantage of inner cities», Harvard Business Review, pp. 55-71.

Sassen, Saskia (2007), Una sociología de la globalización, Buenos Aires, Katz Editores.

Schiavo, Ester y Alejandro Gelfuso (2017), «Ciudades inteligentes y laboratorios ciudadanos. Adjetivando el derecho a la ciudad», en Daniel Hiernaux y Carmen Imelda González (comps.), La ciudad latinoamericana a debate. Perspectivas teórica, Querétaro, Universidad Autónoma de Querétaro, pp. 125-154.

Smith, Neil (1996), The new urban frontier; gentrification and the revanchist city, Londres, Routledge.

Santana Rivas, Luis Daniel y Ángela Milena Alzate Navarro (2017), «Las teorías críticas sobre la producción inmobiliaria: dos perspectivas metromarxistas y una agenda teórica», en Daniel Hiernaux y Carmen Imelda González (comps.), La ciudad latinoamericana a debate. Perspectivas teóricas. Querétaro, Universidad Autónoma de Querétaro, pp. 155-192. 